\title{
Peripheral Uric Acid as a Biomarker in Individuals With Bipolar Disorder: A Case Control Study
}

Zhe Lu

Peking University Sixth Hospital

Yingtan Wang

Jining Medical University

Guanglei Xun ( $\sim$ xungl2019@163.com)

Shandong Mental Health Center, 49\# Wenhua Eastern Road, Jinan 250014, China.

\section{Research Article}

Keywords: Bipolar disorder, Unipolar disorder, Uric acid, Biomarker

Posted Date: January 5th, 2021

DOI: https://doi.org/10.21203/rs.3.rs-137483/v1

License: () (i) This work is licensed under a Creative Commons Attribution 4.0 International License. Read Full License 


\section{Abstract}

Background: At present, no well-established biomarkers were ever found to distinguish unipolar disorder (UD) and bipolar disorder (BD). This study aimed to explore whether uric acid (UA) could be a biomarker to distinguish UD and BD.

Methods: Peripheral UA of 119 patients with BD in acute stage (AS) and 77 in remission stage (RS), and 95 patients with UD in AS and 61 in RS were measured, so were 180 healthy controls. Differences in continuous variables among groups were assessed by the independent samples t-test and one-way analysis of variance. The chi-square test was applied to categorical data such as gender.

Results: UA levels in BD group were higher than UD and HC groups regardless of the AS or RS, while differences in UA levels between UD group and HC group were not significant. Differences of UA levels between BD-M (bipolar mania/hypomania) and BD-D (bipolar depression) subgroups were not significant, and UA levels of BD-M and BD-D subgroups were higher than UD and HC groups. Only in UD group, UA levels of drug-use subgroup were higher than drugnaïve/free subgroup, but differences disappeared when analyzed stratified by sex; whether in drug-use or drug-naïve/free subgroup, differences of UA levels between BD-M and BD-D groups were not significant.

Conclusion: The study suggests UA levels may be a biomarker of BD to distinguish from UD.

\section{Background}

Bipolar disorder (BD) is a serious mental disorder with a low diagnosis rate result from the onset of BD is often characterized by a depressive episode, which is similar in presentation to unipolar depression (UD) [1]. Due to misdiagnosis, inappropriate treatment with antidepressants without concomitant mood stabilizers results in switching to mania or hypomania and repeated attacks of depression [2]. A recent study showed that family history of BD, early age at onset of the first depressive episode ( $\$ 25$ years), postpartum depressive episodes, rapid onset of depressive episodes, worse response to antidepressants and the presence of psychotic symptoms or atypical depressive symptoms might be the most consistent clinical predictors of BD [3]. However, no laboratory or imaging marker is identified to allow for a diagnosis of BD or distinguishing between BD and UD.

The purinergic system is a critical neurotransmitter system with the end product of Uric acid (UA), which involves the occurrence and development of mental illness [4]. It has been proved that increased levels of UA are associated with the accelerated purinergic transformation [5]. UA acts on presynaptic and postsynaptic neurons and specific receptors in the glial cell membrane that can affect other neurotransmitters' activities involved in the pathophysiological process of mood disorders, including dopamine, gamma-aminobutyric acid, glutamate and serotonin [6].

In the late 19th century, researchers found that some patients with gout and hyperuricemia suffered from mood disorders and were relieved after receiving lithium treatment. Since then, the relation between UA and mood disorders has raised the hypothesis of purinergic system dysfunction [7]. Recent studies showed that the highest UA levels were observed in patients with BD compared with other mental disorders and healthy controls (HC) [8-11], and elevated UA levels were associated with impulsivity, excitatory behavior, irritability, hyperthymia temperament and severe manic symptoms [6, 12]. While the lowest UA levels were observed in patients with UD, suggesting that UA may be a potential biomarker for distinguishing between BD and UD. Besides, patients with BD have an increased risk of gout [13], while allopurinol, an inhibitor of xanthine oxidase used to treat and prevent gout, can be used as an add-on therapy for patients with BD to reduce manic symptoms [14]. Some studies also implied that compared with bipolar depression and remission, the highest UA levels were observed in the manic episode, indicating that UA may be a status marker of manic episodes rather than a trait marker [15-17]. However, similar results were not detected in similar studies. Studies by Salvadore G et al. and Gültekin BK et al. showed that UA levels were higher in patients with BD than in healthy controls but not associated with the severity of mania. Furthermore, some studies showed there were no statistically significant differences in UA levels between BD and UD, neither did to healthy controls [18-20].

Previous studies on UA of patients with BD and UD are limited and conflicting. Therefore, whether UA could be a biomarker of BD remains to be further studied. The present study aimed to evaluate whether UA could be a potential biomarker to distinguish between BD and UD.

\section{Methods}

\section{Subjects and participants}

The study protocol was approved by the Clinical Research Ethics Committee of Shandong Mental Health Center and is compliant with the Code of Ethics of the World Medical Association (Declaration of Helsinki). Informed written consent was obtained from all participants or their legal guardians after a complete and extensive description.

The study was conducted at Shandong Mental Health Center, form May 2018 to May 2019, inpatients and outpatients aged from 18-60 years with the Diagnostic and Statistical Manual of Mental Disorders, fifth edition (DSM-5) diagnosis of BD or UD in the acute stage (AS) or remission stage (RS) were recruited. Furthermore, healthy individuals with no family history of psychiatric disorders were enrolled in the study as the control group.

The exclusion criteria were as follows: (1) Combined with organic brain diseases or brain trauma. (2) Hypertension, diabetes, gout or liver, kidney, biliary, and other physical diseases or abnormal renal and liver function. (3) Combined with other mental disorders. (4) Positive in urine pregnancy test or lactating females. (5) Modified electroconvulsive therapy treatment within 4 weeks, or long-acting antipsychotics treatment within 6 months; (6) Taking antioxidants or neurotrophic drugs within 12 weeks before and during enrollment.

\section{Measurements of serum UA levels}


Serum UA levels test as part of routine blood checks was performed during the inpatient stays and the regular return visit of outpatients, while serum UA levels test of healthy individuals in this study was performed after enrollment. The assay was prepared as follows: five milliliters of fasting venous blood samples were drawn from all participants. According to the manufacturer's instructions, serum levels of UA were detected by Roche Cobas C702 automatic biochemical analyzer (Swiss Roche Diagnostics Co., Ltd.). In Shandong Mental Health Center, the normal range of serum UA values has been standardized as 208-428 $\mu \mathrm{mol} / \mathrm{L}$ in males and $155-357 \mu \mathrm{mol} / \mathrm{L}$ in females.

\section{Statistical analysis}

The results were expressed as mean \pm standard deviation (Mean \pm SD). Differences in continuous variables among groups were assessed by the independent samples t-test and one-way analysis of variance. The chi-square test was applied to categorical data such as gender. $P$-values $<0.05$ were considered statistically significant.

\section{Results}

\section{Demographic and clinical data}

The study included 119 BD patients in AS and 77 in RS and included 95 UD patients in AS and 61 in RS as well as 180 subjects in the HC group. Differences of sex among 3 groups were not significant (AS, $c 2=0.837, P=0.658$; RS, $c 2=0.326, P=0.850$ ). There were significant differences in age (AS, $F=10.786, P<0.001$; $\mathrm{RS}, F=5.131, P=0.006)$ among $\mathrm{BD}, \mathrm{UD}$ and HC groups. Significant differences were also found in duration of illness (AS, $t=2.195, P=0.029 ; \mathrm{RS}, t=2.179$, $P=0.030$ ). The differences in smoking history (AS, $c 2=0.192, P=0.661 ; \mathrm{RS}, c 2=0.001, P=0.982)$ and family history (AS, $c 2=1.042, P=0.307 ; \mathrm{RS}, c 2=0.615$, $P=0.433$ ) between BD group and UD group were not significant. Additional information about demographics and clinical characteristics is presented in Table 1.

\section{Differences in UA levels among BD, UD, and HC group in AS}

There were significant differences in UA levels among three groups. Post-hoc analysis showed that UA levels of the BD group were higher than UD ( $P<0.001)$ and $\mathrm{HC}(P<0.001)$ group, while differences in UA levels between UD and HC group were not significant $(P=0.453)$. Similar results were detected when analyzing separately by sex. (Table 2, Figure 1)

Afterward, the BD group was divided into bipolar mania/hypomania (BD-M, $n=64$ ) subgroup and bipolar depression (BD-D, $n=55)$ subgroup to be compared with the UD group and HC group. There were significant differences among the four groups. The post-hoc test showed that differences of UA levels between BD-M subgroup and BD-D subgroup were not significant ( $P=0.213)$, as well as between UD group and HC group ( $P=0.895)$; UA levels of BD-M (UD, $P<0.001 ; \mathrm{HC}$, $P<0.001)$ and BD-D (UD, $P<0.001 ; \mathrm{HC}, P=0.003)$ subgroups were higher than UD group and HC group. When analyzed stratified by sex, similar results were detected in female patients, there was no significant difference on UA levels between subgroup BD-M and BD-D subgroup, so did to UD group and HC group; UA levels of BD-M (UD, $P<0.001 ; \mathrm{HC}, P<0.001)$ and BD-D (UD, $P=0.039 ; \mathrm{HC}, P=0.048)$ subgroups were higher than UD group and HC group. In male patients, the differences of UA levels between BD-D group and HC group were not significant, so did to UD group and HC group; UA levels of BD-M subgroup were higher than the other groups (BD-D, $P<0.001 ; \mathrm{UD}, P<0.001 ; \mathrm{HC}, P<0.001$ ), UA levels of BD-D subgroup were higher than UD group ( $P=0.028)$. (Table 2, Figure 1 )

\section{Differences in UA levels among BD, UD and HC group in RS}

Significant differences in UA levels were spotted among three groups. The post-hoc test showed that UA levels of the BD group were higher than UD ( $P<0.001)$ and $\mathrm{HC}(P<0.001)$ group, while differences in UA levels between UD and HC group were not significant. The similar results were detected when analyzed stratified by sex. (Table 3, Figure 1)

\section{Effects of treatment on UA levels}

\section{Drug-use subgroup vs. drug-naïve/free subgroup}

Patients were divided into drug-use subgroup and drug-naïve/free subgroup (mania and depression unmedicated first episode or no treatment was used within eight weeks). Only in the UD group, there were significant differences in UA levels between drug-use subgroup and drug-naïve/free subgroup, and the differences disappeared when analyzed stratified by sex. (Table 4)

\section{BD-M vs. BD-D vs. UD in the drug-use subgroup}

In the drug-use subgroup, the differences among 3 groups were significant $(F=10.547, P \llbracket 0.001)$, the post-hoc test showed there were no significant differences in UA levels between BD-M and BD-D ( $P=0.846)$ groups, while UA levels of both groups were higher than UD group (BD-M vs. UD, $P<0.001 ; B D-D$ vs. UD, $P=0.012$ ). When analyzed stratified by sex, no matter in male patients or female patients, UA levels of BD-M group were higher than UD group ( $P<0.001)$, there were no significant differences in UA levels between BD-M group and BD-D group as well as BD-D and UD group. (Figure 2)

\section{BD-M vs. BD-D vs. UD in drug-naïve/free subgroup}

In drug-naïve/free subgroup, the differences among 3 groups were significant ( $F=11.632, P \Downarrow 0.001)$, there were no significant differences in UA levels between BD-M and BD-D ( $P=0.301)$ groups, but UA levels of both groups were higher than UD (BD-M vs. UD, $P<0.001 ; B D-D$ vs. UD, $P=0.021)$ group. In male patients, UA levels of BD-M group were higher than BD-D group ( $P=0.025)$ and UD group $(P=0.001)$, however, the differences of UA levels between BD-D group and UD 
group were not significant. In female patients, UA levels of BD-M group were higher than UD group $(P<0.001)$, there were no significant differences in UA levels between BD-M group and BD-D group as well as BD-D and UD group. (Figure 2)

\section{Discussion}

The purinergic system is involved in neurodevelopment and pathophysiological processes of psychotic disorders, such as the process of genesis, differentiation on neurocyte and inflammation of neuro-glial cell, and so on [21-24]. Purinergic receptors can be divided into P1 and P2 receptors according to their biochemical and pharmacological properties [25]. P1 receptors can regular plasticity of synapse and the release of neurotransmitters [23, 24, 26, 27], while P2 receptors are closely related to embryonic neural development [28]. The dysfunction of the purinergic system result from any causes may lead to psychotic disorders. UA, as the end product of the purinergic system, is in connection with some physiological functions, including sleep, motor, cognitive function, appetite, and social activities, as well as the pathophysiology of mood disorders [6,12]. Additionally, UA is also related to specific traits, including driving and disinhibition, which is very common in BD. It is also noticed that the peripheral UA levels are consistent with that in the central nervous system [29, 30].

In the study, UA levels in the BD group were higher than UD and HC groups, whether in AS or RS, which suggested that UA levels may be a potential biomarker to distinguish between BD and UD. Nevertheless, a recent study indicated that UA levels in UD were lower than HC; a possible reason was the heterogeneity of subjects in the UD group because the UA diagnosis is only based on clinical symptoms at present while some patients with BD often begin with depression. It was further confirmed by a recent study that the higher UA levels might be a predictor of BD [31]. The previous study showed that sex was an important factor that could affect UA levels [19], but the study analyzed separately by sex and got similar results. Beyond that, UA is also a selective antioxidant whose level is considered as a marker of oxidative stress, and results in this study indicated that patients with BD might have a higher oxidative stress level. Moreover, the study divided acute patients with BD into BD-M and BD-D subgroups, with results showing that differences of UA levels between BD-M subgroup and BD-D subgroup were not significant, and UA levels of both subgroups were higher than UD group. When analyzed stratified by sex, significant differences in UA levels between male patients of BD-M and BD-D subgroups were spotted, but the differences were not significant in female patients, which suggested that male patients might be more susceptible to different stages of illness.

In order to detect the effects of treatment on UA levels, the study divided the acute patients into drug-use and drug-naïve/free subgroups. It was observed that UA levels of the drug-use subgroup were higher than drug-naïve/free subgroup only in the UD group, and the differences were not significant when analyzing stratified by sex, which suggested that UA might be a steady biomarker to distinguish BD and UD. Moreover, UA levels in male patients of the BD-M subgroup were higher than the BD-D subgroup, whether in drug-use or drug-naïve/free subgroup, and the differences in female patients were not significant, which confirmed that male patients might be more susceptible to different stages of illness.

There are a few limitations to this study. Firstly, the study did not collect full demographic and clinical data, such as attack times, previous medication use, and so on. More else, diet is also an affecting factor to UA levels, but this study did not strictly control the diet. Secondly, biochemical indicators, like hepatorenal function and indexes of glycometabolism and lipometabolism, were not collected. Mediation analysis indicated that metabolic syndrome, triglyceride, and abdominal perimeter could affect UA levels, although it could not fully explain the correlation between UA and BD [8]. Thirdly, we did not evaluate the severity of the disease. A previous study showed that UA levels were positively correlated with the severity of mania [9], but recent studies indicated that there was no significant correlation between UA and severity of mania [18,32], which is calling for more strictly designed prospective studies to explore the relation between UA and severity of the disease. Finally, although the study divided acute patients into drug-use and drug-naïve/free subgroup, the effect of different kinds of mood stabilizers on UA levels are diverse, such as lithium [33] and carbamazepine may decrease UA levels of BD patients, while valproates seemly have the opposite effect [34], and the effect of antidepressants, physiotherapeutic and psychotherapy on UA levels were not yet discussed.

\section{Conclusions}

This study observed that UA levels in BD were higher than UD and HC. Antimanic therapy or antidepressant therapy makes little difference in BD patients. In conclusion, the present study indicates that UA levels may be a steady biomarker of BD to distinguish from UD and provide further evidence on the relation between the purinergic system and pathogenesis of $\mathrm{BD}$. In the future, a strict-design, larger-sample prospective study is required to confirm this conclusion.

\section{Abbreviations}

UA, uric acid; BD-M, mania/hypomania; BD-D, bipolar depression; UD, unipolar disorder; HC, healthy control; BD-M, bipolar mania/hypomania; BD-D, bipolar depression; AS, acute stag; RS, remission stage; DSM-5, Diagnostic and Statistical Manual of Mental Disorders, fifth edition.

\section{Declarations}

\section{Ethics approval and consent to participate}

The study protocol was approved by the Clinical Research Ethics Committee of Shandong Mental Health Center and is compliant with the Code of Ethics of the World Medical Association (Declaration of Helsinki). Informed written consent was obtained from all participants or their legal guardians after a complete and extensive description.

\section{Consent for publication}

Not applicable 


\section{Availability of data and materials}

The datasets used and/or analyzed during the current study are available from the corresponding author on reasonable request.

\section{Competing interests}

The authors declare that they have no competing interests.

\section{Funding}

This research did not receive any specific grant from funding agencies in the public, commercial, or not-for-profit sectors.

\section{Authors' contributions}

ZL and GLX designed the study, carried out the experiments and analyzed the data statistically. ZL and YTW performed data collection. ZL wrote the manuscript. All authors read and approved the final manuscript.

\section{Acknowledgments}

The authors would like to thank patients in Shandong Mental Health Center and healthy individuals in this study for their support and participation.

\section{References}

1. Tondo L, Visioli C, Preti A, Baldessarini RJ. Bipolar disorders following initial depression: Modeling predictive clinical factors. J Affect Disord. 2014;167(10):44-9.

2. Liu B, Zhang Y, Fang H, Liu J, Liu T, Li L. Efficacy and safety of long-term antidepressant treatment for bipolar disorders - A meta-analysis of randomized controlled trials. J Affect Disord. 2017;223:41-8.

3. Stahl SM, Morrissette DA, Faedda G, Fava M, Goldberg JF, Keck PE, et al. Guidelines for the recognition and management of mixed depression. CNS Spectr. 2017;22(2):203-19.

4. Cheffer A, Castillo ARG, Correa-Velloso J, Goncalves MCB, Naaldijk Y, Nascimento IC, et al. Purinergic system in psychiatric diseases. Mol Psychiatry. 2018;23(1):94-106.

5. Burnstock G. Purinergic signalling and disorders of the central nervous system. Nature Reviews Drug Discovery. 2008;7(7):575-90.

6. Machado-Vieira R, Lara DR, Souza DO, Kapczinski F. Purinergic dysfunction in mania: an integrative model. Medical Hypotheses. 2002;58(4):297-304.

7. Ortiz R, Ulrich H, Jr CAZ, Machado-Vieira R. Purinergic system dysfunction in mood disorders: a key target for developing improved therapeutics. Progress in Neuropsychopharmacology Biological Psychiatry. 2015;57:117-31.

8. Bartoli F, Crocamo C, Gennaro GM, Castagna G, Trotta G, Clerici M, et al. Exploring the association between bipolar disorder and uric acid: A mediation analysis. J Psychosom Res. 2016;84:56-9.

9. De Berardis D, Conti CM, Campanella D, Carano A, Di Giuseppe B, Valchera A, et al. Evaluation of plasma antioxidant levels during different phases of illness in adult patients with bipolar disorder. Journal Of Biological Regulators And Homeostatic Agents. 2008;22(3):195-200.

10. Ezzaher A, Mouhamed DH, Mechri A, Neffati F, Douki W, Gaha L, et al. TBARs and non-enzymatic antioxidant parameters in Tunisian bipolar I patients. Immuno-analyse et biologie spécialisée. 2012;27(6):315-24.

11. Wen S, Cheng M, Wang H, Yue J, Wang H, Li G, et al. Serum uric acid levels and the clinical characteristics of depression. Clin Biochem. 2012;45(1-2):4953.

12. Sutin AR, Cutler RG, Camandola S, Uda M, Feldman NH, Cucca F, et al. Impulsivity is Associated with Uric Acid: Evidence from Humans and Mice. Biological Psychiatry. 2014;75(1):31-7.

13. Chung KH, Huang CC, Lin HC. Increased risk of gout among patients with bipolar disorder: a nationwide population-based study. Psychiatry Res. 2010;180(2-3):147-50.

14. Chen AT, Malmstrom T, Nasrallah HA. Allopurinol augmentation in acute mania: A meta-analysis of placebo-controlled trials. Journal of Affective Disorders. 2017;226:245.

15. Muti M, Del Grande C, Musetti L, Marazziti D, Turri M, Cirronis M, et al. Serum uric acid levels and different phases of illness in bipolar I patients treated with lithium. Psychiatry Research. 2015;225(3):604-8.

16. Kesebir S, Süner O, Yaylaci ET, Bayrak A, Turan C. Increased uric acid levels in bipolar disorder: is it trait or state? Biol Regul Homeost Agents. 2013;27(4):981-8.

17. Albert U, De Cori D, Aguglia A, Barbaro F, Bogetto F, Maina G. Increased uric acid levels in bipolar disorder subjects during different phases of illness. J Affect Disord. 2015;173:170-5.

18. Salvadore G, Viale Cl, Luckenbaugh DA, Zanatto VC, Portela LV, Souza DO, et al. Increased uric acid levels in drug-naive subjects with bipolar disorder during a first manic episode. Progress In Neuro-Psychopharmacology \& Biological Psychiatry. 2010;34(6):819-21.

19. Wiener C, Rassier GT, Kaster MP, Jansen K, Pinheiro RT, Klamt F, et al. Gender-based differences in oxidative stress parameters do not underlie the differences in mood disorders susceptibility between sexes. Eur Psychiatry. 2014;29(1):58-63. 
20. Gültekin BK, Kesebir S, Kabak SG, Ergün FF, Tatlidil YE. Are Uric Acid Levels Different from Healthy Subjects in Bipolar Affective Disorder and Schizophrenia?: Relationship Between Clinical Improvement and Episode Severity in Male Patients. Noro Psikiyatri Arsivi. 2014;51(3):229.

21. Burnstock G, Krügel U, Abbracchio MP, Illes P. Purinergic signalling: From normal behaviour to pathological brain function. Progress in Neurobiology. 2011;95(2):229-74.

22. Burnstock G. Introductory overview of purinergic signalling. Frontiers in Bioscience. 2011;3(3):896-900.

23. Cunha RA. Neuroprotection by adenosine in the brain: From A1 receptor activation to A2A receptor blockade. Purinergic Signalling. 2005;1(2):111-34.

24. Fredholm BB, Chen JF, Cunha RA, Svenningsson P, Vaugeois JM. Adenosine and Brain Function. International Review of Neurobiology. 2005;63(1):191270.

25. Fredholm BB, Abbracchio MP, Burnstock G, Dubyak GR, Harden TK, Jacobson KA, et al. Towards a revised nomenclature for P1 and P2 receptors. Trends in Pharmacological Sciences. 1997;18(3):79-82.

26. Campbell, Nicholas G, Lindler, Kathryn M, Hewlett, William A, et al. Rare coding variants of the adenosine A3 receptor are increased in;autism: on the trail of the serotonin transporter regulome. Molecular Autism,4,1. 2013;4(1):28-.

27. Dennis SH, Jaafari N, Cimarosti H, Hanley JG, Henley JM, Mellor JR. Oxygen/Glucose Deprivation Induces a Reduction in Synaptic AMPA Receptors on Hippocampal CA3 Neurons Mediated by mGluR1 and Adenosine A3 Receptors. Journal of Neuroscience. 2011;31(33):11941-52.

28. Oliveira Á, Illes P, Ulrich H. Purinergic receptors in embryonic and adult neurogenesis. Neuropharmacology. 2016;104:272-81.

29. Machado-Vieira R. Purinergic system in the treatment of bipolar disorder: uric acid levels as a screening test in mania. Journal of Clinical Psychopharmacology. 2012;32(5):735-6.

30. Bowman GL, Shannon J, Frei BKaye JA, Quinn JF. Uric acid as a CNS antioxidant. Journal of Alzheimers Disease. 2010;19(4):1331-6.

31. Dos Santos Oliveira PM, Santos V, Coroa M, Ribeiro J, Madeira N. Serum uric acid as a predictor of bipolarity in individuals with a major depressive episode. Bipolar Disord. 2019;21(3):235-43.

32. Bartoli F, Crocamo C, Dakanalis A, Brosio E, Miotto A, Capuzzi E, et al. Purinergic system dysfunctions in subjects with bipolar disorder: A comparative cross-sectional study. Compr Psychiatry. 2017;73:1-6.

33. A Anumonye MBL, M.SC. Edin., D.P.M, F Knight MBD, D.P.M. Uric-acid metabolism in manic-depressive illness and during lithium therapy. Lancet. 1968;291(7555):1290-3.

34. Ring HA, Heller AJ, Marshall WJ, Johnson AL, Reynolds EH. Plasma uric acid in patients receiving anticonvulsant monotherapy. Epilepsy Res. $1991 ; 8(3): 241-4$

\section{Tables}

Table 1. Demographic characteristic and clinical data of participants.

\begin{tabular}{|c|c|c|c|c|c|}
\hline & \multicolumn{2}{|l|}{$\mathrm{BD}$} & \multicolumn{2}{|l|}{ UD } & \multirow[t]{2}{*}{$\mathrm{HC}$} \\
\hline & AS & RS & AS & RS & \\
\hline & $\mathrm{n}=119$ & $\mathrm{n}=77$ & $\mathrm{n}=95$ & $n=61$ & $n=180$ \\
\hline Gender (male/female) & $61 / 58$ & $37 / 40$ & $43 / 52$ & $28 / 33$ & $90 / 90$ \\
\hline $\begin{array}{l}\text { Age } \\
\text { (years) }\end{array}$ & $31.91 \pm 11.68$ & $32.75 \pm 11.92$ & $37.63 \pm 13.41$ & $36.03 \pm 13.87$ & $37.48 \pm 9.04$ \\
\hline Smokers/Non-smokers & $21 / 98$ & $14 / 63$ & $19 / 76$ & $11 / 50$ & $\mathrm{~N}$ \\
\hline Family history (positive/negative) & $22 / 97$ & $17 / 60$ & $23 / 72$ & $17 / 44$ & $\mathrm{~N}$ \\
\hline Duration of illness $₫$ months $\rrbracket$ & $77.10 \pm 74.70$ & $83.17 \pm 83.83$ & $53.82 \pm 80.01$ & $51.74 \pm 82.99$ & $\mathrm{~N}$ \\
\hline
\end{tabular}

BD, bipolar disorder; UD, unipolar disorder; $\mathrm{HC}$, healthy control; AS, acute stag; RS, remission stage.

Table 2. UA (uric acid) levels of participants in AS ( $\mu \mathrm{mol} / \mathrm{L})$ 


\begin{tabular}{|c|c|c|c|c|c|c|c|c|c|c|c|c|}
\hline & \multicolumn{3}{|l|}{ BD } & \multicolumn{3}{|l|}{ BD-M } & \multicolumn{3}{|l|}{ BD-D } & \multicolumn{3}{|l|}{ UD } \\
\hline & & male & female & & male & female & & male & female & & male & femi \\
\hline & $n=119$ & $n=61$ & $n=58$ & $n=64$ & $n=27$ & $n=37$ & $\mathrm{n}=55$ & $n=34$ & $\mathrm{n}=21$ & $n=95$ & $n=43$ & $\mathrm{n}=5$; \\
\hline $\mathrm{U}$ & $354.02 \pm$ & $394.10 \pm 87.61$ & $311.86 \pm 68.54$ & 367.84 & $431.63 \pm$ & $321.30 \pm 68.55$ & 337.93 & $364.29 \pm$ & $295.24 \pm 66.91$ & 282.13 & $321.95 \pm$ & 249. \\
\hline \multirow[t]{2}{*}{ A } & 88.75 & & & \pm & 84.29 & & \pm & 79.30 & & \pm & 60.59 & \\
\hline & & & & 92.92 & & & 81.54 & & & 77.98 & & \\
\hline
\end{tabular}

UA, uric acid; BD-M, mania/hypomania; BD-D, bipolar depression; UD, unipolar disorder; HC, healthy control.

$F 1$ and $P 1$ were the statistic of comparison among BD, UD and HC; $F 2$ and $P 2$ were the statistic of comparison among $B D, U D$ and $H C$ in males; $F 3$ and $P 2$ were the statistic of comparison among BD, UD and $\mathrm{HC}$ in females; $F 4$ and $P 4$ were the statistic of comparison among BD-M, BD-D, UD and HC; F5 and P5 were the statistic of comparison among BD-M, BD-D, UD and HC in males; $F 6$ and $P 6$ were the statistic of comparison among BD-M, BD-D, UD and HC in females.

Table 3. UA (uric acid) levels of participants in RS ( $\mu \mathrm{mol} / \mathrm{L}$ )

\begin{tabular}{|c|c|c|c|c|c|c|c|c|c|c|}
\hline & \multicolumn{3}{|l|}{$\mathrm{BD}$} & \multicolumn{3}{|l|}{ UD } & \multicolumn{3}{|l|}{$\mathrm{HC}$} & \multirow[t]{2}{*}{ F1/F2/F3 } \\
\hline & & male & female & & male & female & & male & female & \\
\hline & $\mathrm{n}=77$ & $n=37$ & $\mathrm{n}=40$ & $n=61$ & $\mathrm{n}=28$ & $n=52$ & $n=180$ & $\mathrm{n}=90$ & $\mathrm{n}=90$ & \\
\hline \multirow[t]{3}{*}{ UA } & $364.17 \pm$ & $401.32 \pm 92.16$ & $329.80 \pm 76.25$ & 295.84 & $352.96 \pm$ & $247.36 \pm 60.70$ & 296.27 & $339.29 \pm$ & $253.24 \pm 54.99$ & 23.315/12.642/24.126 \\
\hline & 91.11 & & & \pm & 46.90 & & \pm & 52.37 & & \\
\hline & & & & 95.96 & & & 68.77 & & & \\
\hline
\end{tabular}

UA, uric acid; BD, bipolar disorder; UD, unipolar disorder; HC, healthy control.

$F 1$ and $P 1$ were the statistic of comparison among BD, UD and HC; $F 2$ and $P 2$ were the statistic of comparison among $\mathrm{BD}, \mathrm{UD}$ and $\mathrm{HC}$ in males; $F 3$ and $P 2$ were the statistic of comparison among $\mathrm{BD}, \mathrm{UD}$ and $\mathrm{HC}$ in females;

Table 4. UA levels of drug-use subgroup and drug-naïve/free subgroup ( $\mu \mathrm{mol} / \mathrm{L})$

\begin{tabular}{|lllll|}
\hline & Drug-use & Drug-naïve/free & $t$ & $P$ \\
\hline BD-M(34/30) & $379.65 \pm 84.67$ & $354.47 \pm 101.24$ & 1.083 & 0.283 \\
\hline Male(17/10) & $426.12 \pm 63.73$ & $441.00 \pm 114.68$ & 0.378 & 0.712 \\
\hline Female(17/20) & $333.18 \pm 78.33$ & $311.20 \pm 59.18$ & 0.971 & 0.338 \\
\hline BD-D(28/27) & $356.86 \pm 87.07$ & $318.30 \pm 71.76$ & 1.789 & 0.079 \\
\hline Male(20/14) & $375.95 \pm 81.83$ & $347.64 \pm 75.30$ & 1.025 & 0.313 \\
\hline Female(8/13) & $309.13 \pm 86.13$ & $286.69 \pm 54.11$ & 0.737 & 0.470 \\
\hline UD(50/45) & $299.26 \pm 78.59$ & $263.09 \pm 73.53$ & 2.309 & 0.023 \\
\hline Male(26/17) & $331.19 \pm 60.15$ & $307.82 \pm 60.28$ & 1.245 & 0.220 \\
\hline Female(24/28) & $264.67 \pm 82.62$ & $235.93 \pm 68.07$ & 1.375 & 0.175 \\
\hline
\end{tabular}

UA, uric acid; BD-M, mania/hypomania; BD-D, bipolar depression; UD, unipolar disorder.

\section{Figures}




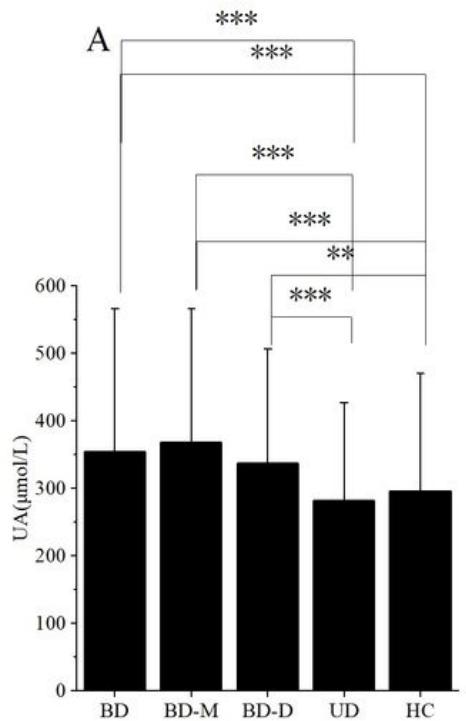

D

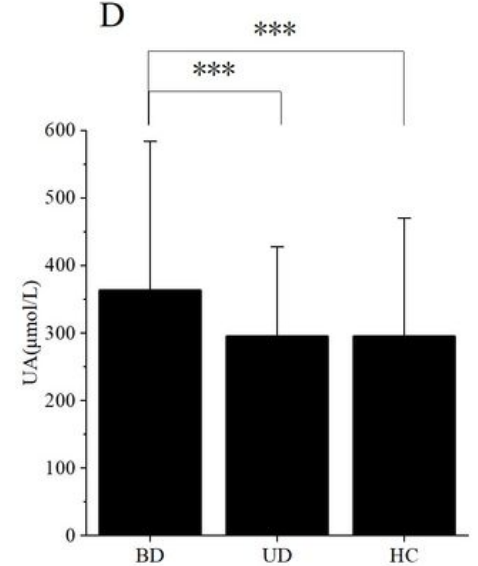

B

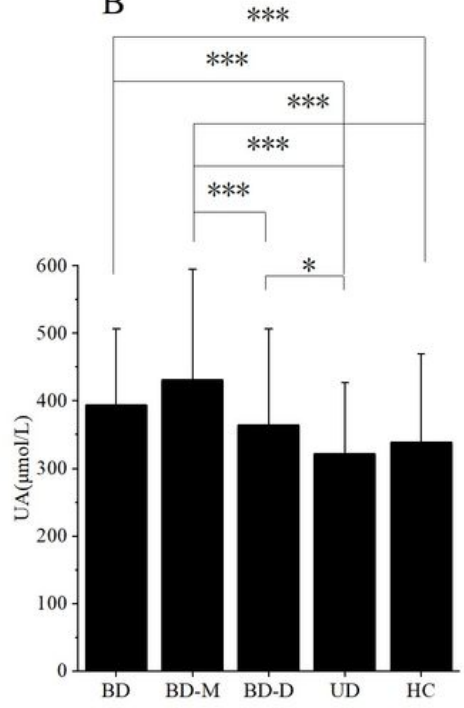

$\mathrm{E}$

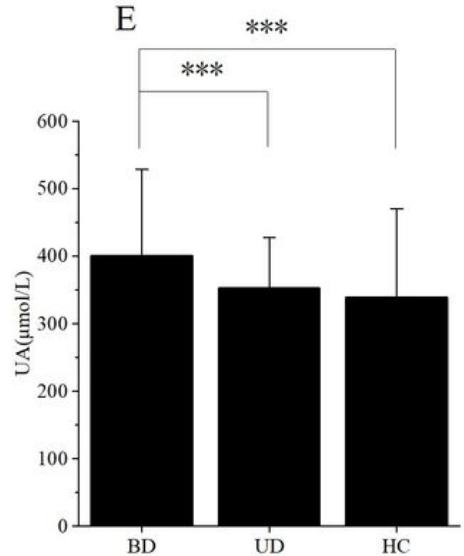

$\mathrm{C}$
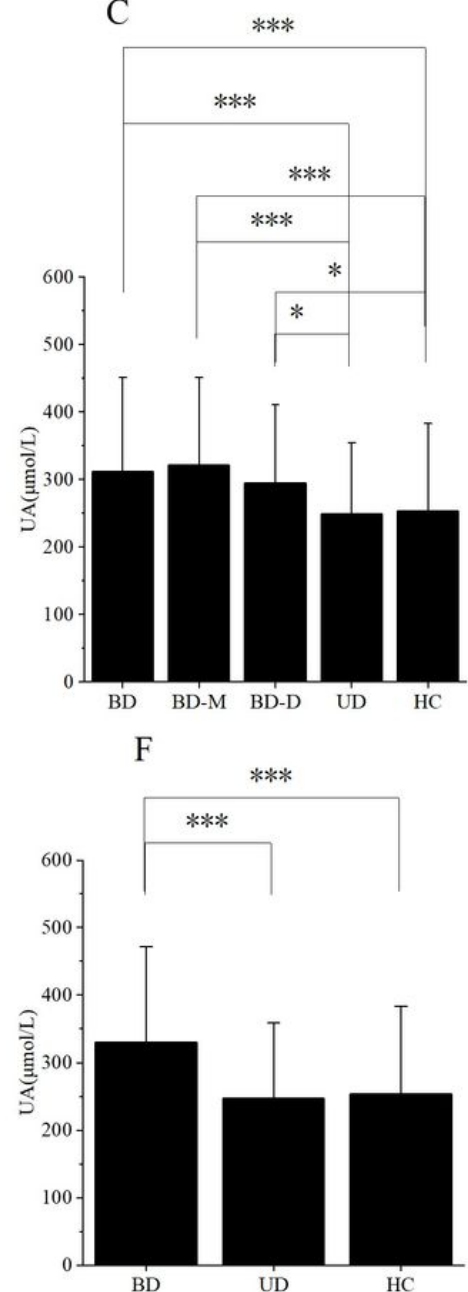

Figure 1

UA levels of participants A, UA (uric acid) levels of all participants in AS (acute stage); B, UA levels of male participants in AS; C, UA levels of female participants in AS; D, UA levels of all participants in RS (remission stage); E, UA levels of male participants in RS; F, UA levels of female participants in RS. 

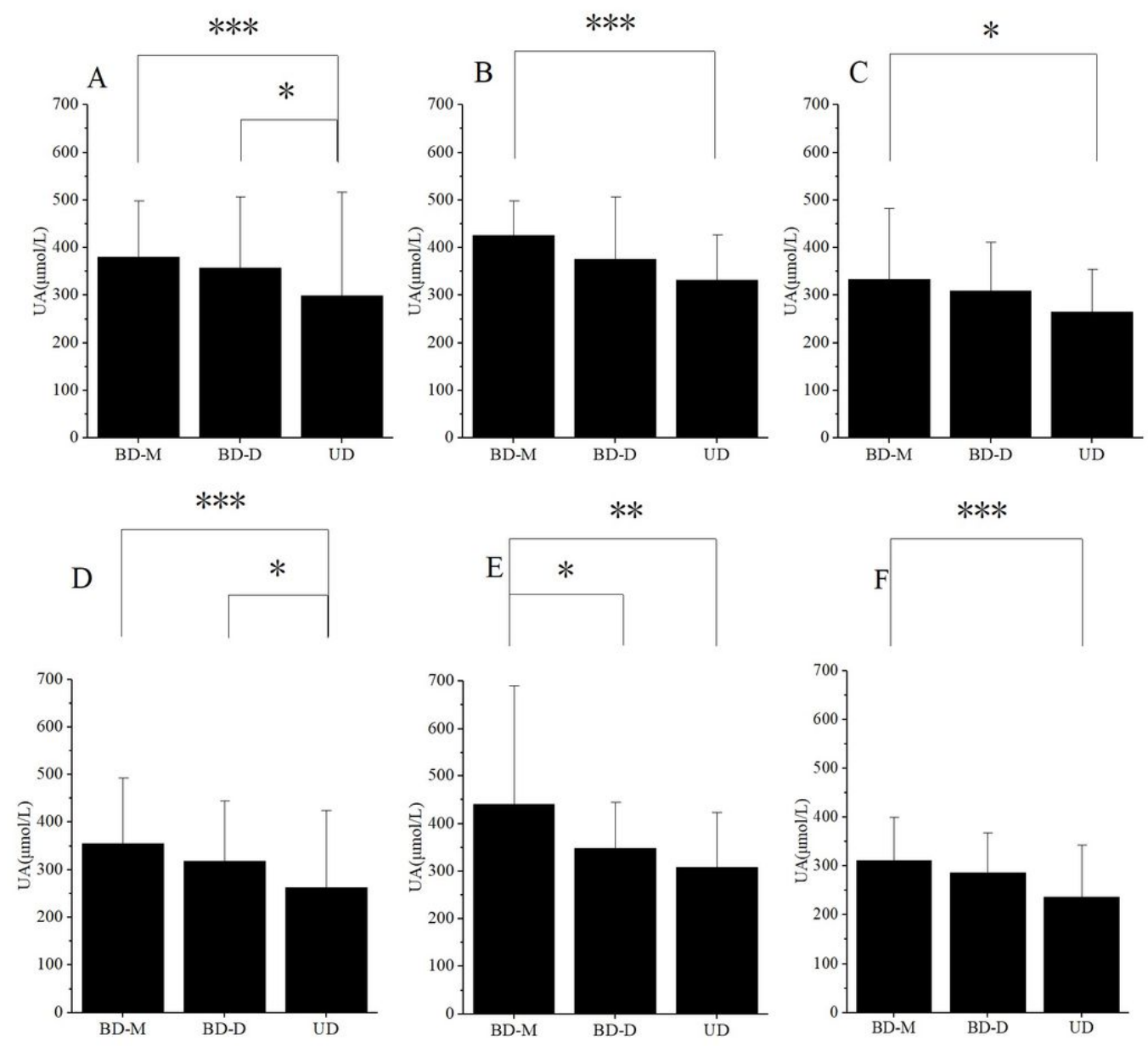

\section{Figure 2}

UA levels of participants A, UA (Uric Acid) levels of all participants in drug-use group; B, UA levels of male participants in drug-use group; C, UA levels of female participants in drug-use group; D UA levels of all participants in drug-native/free group; E, UA levels of male participants in drug-native/free group; F, UA levels of female participants in drug-native/free group. 